Supporting Information

\title{
Mind the Mines: Lapieite Minerals with Ultra-Low Lattice Thermal Conductivity and High Power Factor for Thermoelectricity
}

\author{
Shima Shahabfar ${ }^{1}$ and S. Shahab Naghavi ${ }^{1 *}$ \\ ${ }^{1}$ Department of Physical and Computational Chemistry, Shahid Beheshti University, Tehran \\ 1983969411, Iran \\ E-mail: s_naghavi@sbu.ac.ir
}


a)
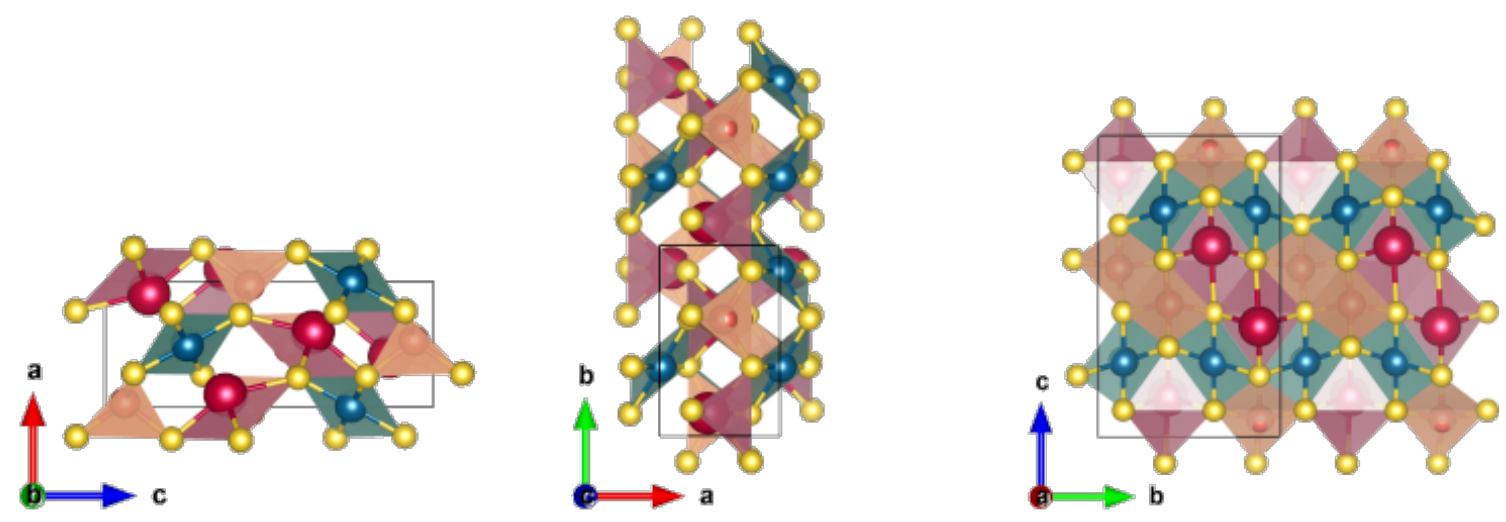

b)

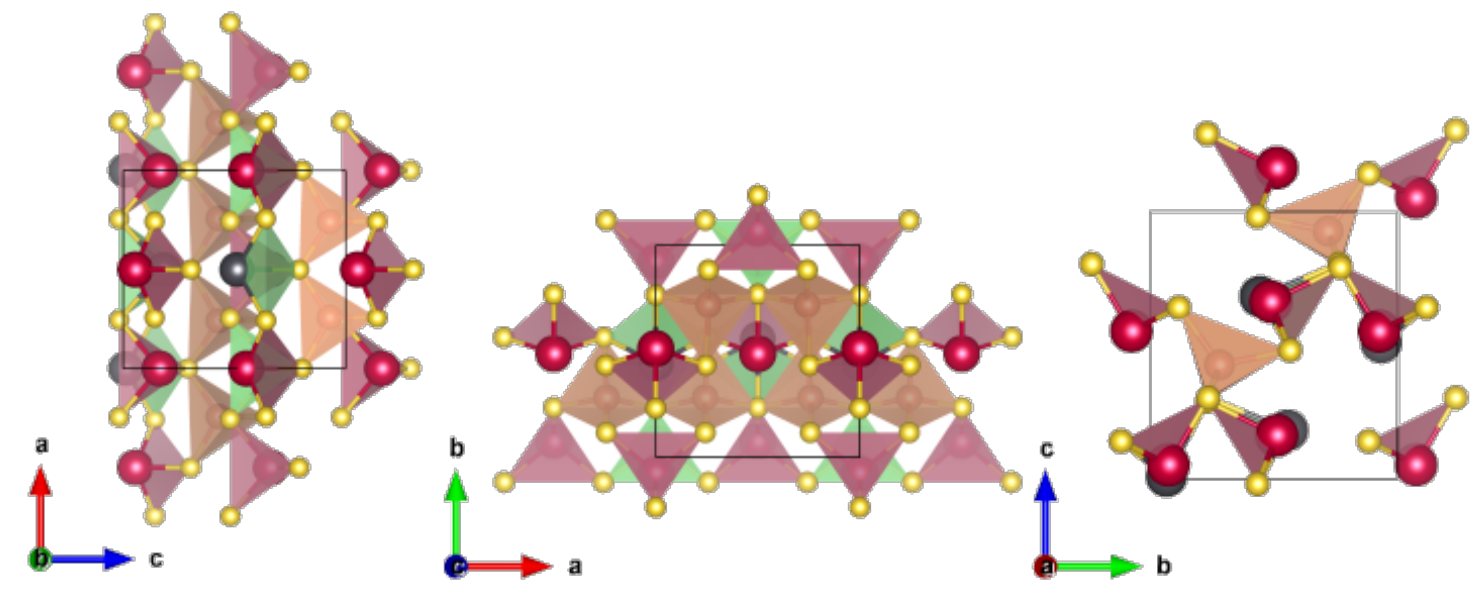

c)
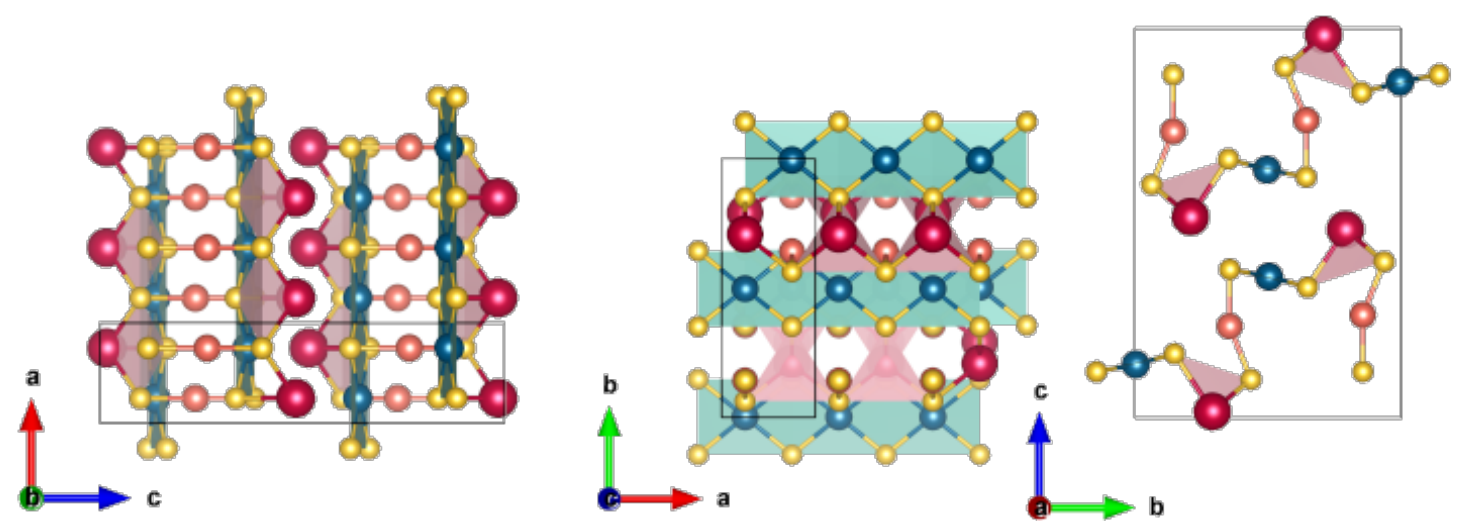

Fig. S1: (a) $P 2_{1} 2_{1} 2_{1}$, (b) $P n 2_{1} m$, and (c) $P n m a$ structures from different angles. 
Table S 1: Space group (SG), band gap energy ( $\left.E_{\text {gap }}\right)$, crystallographic information and calculated power factors $(P F)$.

\begin{tabular}{|c|c|c|c|c|c|c|c|c|c|c|c|c|c|}
\hline Family & Compounds & Name & SG & $E_{\text {gap }}(\mathrm{eV})$ & $a$ & $b$ & $c$ & $P F_{x x}^{\text {hol }}$ & $P F_{y y}^{\text {hol }}$ & $P F_{z z}^{\text {hol }}$ & $P F_{x x}^{\text {ele }}$ & $P F_{y y}^{\text {ele }}$ & $P F_{z z}^{\text {ele }}$ \\
\hline \multirow{12}{*}{ } & $\mathrm{NiCuSbS}_{3}$ & Lapieite & $P 2_{1} 2_{1} 2_{1}$ & 0.350 & 4.925 & 7.511 & 12.354 & 1.45 & 1.80 & 1.93 & 0.76 & 0.77 & 1.92 \\
\hline & & & & & & & & 1.30 & 1.75 & 1.84 & 0.77 & 0.77 & 1.85 \\
\hline & $\mathrm{NiCuBiS}_{3}$ & Mückeite & $P 2{ }_{1} 2_{1} 2_{1}$ & 0.283 & 4.932 & 7.628 & 12.402 & 2.39 & 1.41 & 1.69 & 0.67 & 0.71 & 1.60 \\
\hline & & & & & & & & 2.21 & 1.46 & 1.54 & 0.63 & 0.71 & 1.53 \\
\hline & $\mathrm{PtCuBiS}_{3}$ & Lisiguangite & $P 2{ }_{1} 2_{1} 2_{1}$ & 0.477 & 5.004 & 7.829 & 12.649 & 2.29 & 1.18 & 1.65 & 1.21 & 0.84 & 2.00 \\
\hline & & & & & & & & 1.68 & 1.33 & 1.21 & 0.68 & 0.50 & 0.84 \\
\hline & $\mathrm{PdCuBiS}_{3}$ & Malyshevite & Pnma & 0.665 & 3.687 & 10.155 & 14.853 & 0.83 & 0.42 & 0.74 & 2.05 & 0.70 & 0.73 \\
\hline & & & & & & & & 0.83 & 0.42 & 0.71 & 2.59 & 0.39 & 0.56 \\
\hline & $\mathrm{PdCuBiS}_{3}$ & Synthetic & $P 2{ }_{1} 2_{1} 2_{1}$ & 0.501 & 5.023 & 7.777 & 12.778 & 2.48 & 1.58 & 2.89 & 0.57 & 0.53 & 1.90 \\
\hline & & & & & & & & 2.25 & 1.63 & 2.66 & 0.55 & 0.61 & 0.90 \\
\hline & $\mathrm{PdCuBiSe}_{3}$ & Roterbärite & $P 2{ }_{1} 2_{1} 2_{1}$ & 0.311 & 5.023 & 7.777 & 12.778 & 2.00 & 1.74 & 2.98 & 0.60 & 0.62 & 1.66 \\
\hline & & & & & & & & 1.68 & 1.62 & 2.53 & 0.67 & 0.82 & 0.61 \\
\hline \multirow{8}{*}{ مُّ } & $\mathrm{PbCuSbS}_{3}$ & Bournonite & $P n 2{ }_{1} m$ & 0.697 & 7.861 & 8.283 & 8.991 & 1.64 & 0.14 & 0.26 & 1.01 & 0.20 & 0.70 \\
\hline & & & & & & & & 1.67 & 0.14 & 0.24 & 0.41 & 0.10 & 0.33 \\
\hline & $\mathrm{PbCuAsS}_{3}$ & Seligmannite & $P n 2_{1} m$ & 0.812 & 7.696 & 8.185 & 8.998 & 1.40 & 0.15 & 0.27 & 0.92 & 0.19 & 0.47 \\
\hline & & & & & & & & 1.41 & 0.14 & 0.26 & 0.38 & 0.13 & 0.34 \\
\hline & $\mathrm{PbCuBiS}_{3}$ & Cerromojonite & $P n 2_{1} m$ & 0.393 & 7.941 & 8.258 & 8.920 & 1.87 & 0.14 & 1.86 & 0.59 & 0.14 & 0.40 \\
\hline & & & & & & & & 2.02 & 0.15 & 1.54 & 0.30 & 0.13 & 0.27 \\
\hline & $\mathrm{PbCuBiSe}_{3}$ & Cerromojonite & $P n 2_{1} m$ & 0.109 & 8.250 & 8.604 & 9.170 & 1.63 & 1.17 & 2.40 & 0.49 & 0.15 & 0.31 \\
\hline & & & & & & & & 1.64 & 1.13 & 1.96 & 0.22 & 0.07 & 0.21 \\
\hline \multirow{10}{*}{ 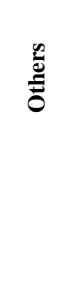 } & $\mathrm{PbCuBiS}_{3}$ & Aikinite & Pnma & 0.601 & 4.054 & 11.304 & 11.896 & 1.13 & 0.52 & 0.72 & 1.36 & 0.86 & 0.57 \\
\hline & & & & & & & & 1.18 & 0.6 & 0.86 & 1.12 & 0.77 & 0.91 \\
\hline & $\mathrm{PbCuBiSe}_{3}$ & & Pnma & 0.415 & 4.193 & 11.703 & 12.559 & 1.11 & 0.78 & 0.96 & 1.47 & 1.05 & 0.79 \\
\hline & & & & & & & & 0.80 & 0.7 & 0.99 & 1.26 & 0.59 & 0.67 \\
\hline & $\mathrm{PdCuBiSe}_{3}$ & & Pnma & 0.474 & 3.845 & 10.000 & 15.849 & 0.48 & 0.23 & 0.88 & 1.32 & 1.31 & 1.14 \\
\hline & & & & & & & & 0.55 & 0.24 & 0.93 & 1.25 & 1.01 & 1.06 \\
\hline & $\mathrm{PtCuBiSe}_{3}$ & & $P 2_{1} 2_{1} 2_{1}$ & 0.272 & 5.129 & 8.151 & 13.381 & 1.48 & 1.23 & 1.44 & 1.14 & 1.89 & 1.01 \\
\hline & & & & & & & & 1.28 & 1.52 & 1.33 & 0.83 & 1.07 & 0.66 \\
\hline & $\mathrm{NiCuBiSe}_{3}$ & & $P 2_{1} 2_{1} 2_{1}$ & 0.149 & 5.071 & 7.995 & 13.111 & 2.16 & 1.52 & 2.26 & 0.59 & 0.6 & 2.22 \\
\hline & & & & & & & & 1.56 & 1.54 & 1.44 & 0.74 & 0.77 & 0.87 \\
\hline \multirow{21}{*}{ 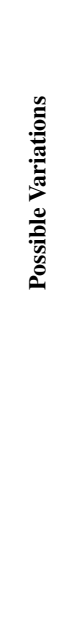 } & $\mathrm{PdCuBiSSe}_{2}$ & & $P 2{ }_{1} 2_{1} 2_{1}$ & 0.332 & 5.088 & 7.989 & 13.299 & 1.39 & 1.26 & 1.25 & 0.54 & 0.58 & 1.27 \\
\hline & & & & & & & & 1.41 & 1.27 & 1.15 & 0.62 & 0.8 & 0.55 \\
\hline & PdCuBiSeSSe & & $P 2{ }_{1} 2_{1} 2_{1}$ & 0.205 & 5.146 & 8.072 & 13.148 & 1.78 & 1.95 & 2.58 & 0.43 & 0.43 & 0.48 \\
\hline & $\mathrm{PdCuBiSe}_{2} \mathrm{~S}$ & & $P 2_{1} 2_{1} 2_{1}$ & 0.471 & 5.101 & 7.991 & 13.306 & 1.71 & 1.26 & 2.7 & 0.84 & 0.58 & 2.38 \\
\hline & & & & & & & & 1.6 & 1.07 & 2.34 & 0.93 & 0.83 & 0.6 \\
\hline & $\mathrm{PdCuBiS}_{2} \mathrm{Se}$ & & $P 2{ }_{1} 2_{1} 2_{1}$ & 0.286 & 5.085 & 7.939 & 12.911 & 1.86 & 1.46 & 2.38 & 0.37 & 0.54 & 1.58 \\
\hline & $\mathrm{PdCuBiSeS}_{2}$ & & $P 2_{1} 2_{1} 2_{1}$ & 0.403 & 5.070 & 7.944 & 12.986 & 1.72 & 1.42 & 1.89 & 0.86 & 0.8 & 1.31 \\
\hline & & & & & & & & 1.76 & 1.39 & 1.85 & 0.97 & 0.86 & 0.71 \\
\hline & PdCuBiSSeS & & $P 2_{1} 2_{1} 2_{1}$ & 0.560 & 5.067 & 7.793 & 13.146 & 1.91 & 1.85 & 3.05 & 0.55 & 0.44 & 0.48 \\
\hline & & & & & & & & 1.57 & 1.67 & 2.42 & 0.62 & 0.58 & 0.64 \\
\hline & $\mathrm{PdAgBiSe}_{3}$ & & $P 2{ }_{1} 2_{1} 2_{1}$ & 0.452 & 5.060 & 8.495 & 14.287 & 1.25 & 0.97 & 1.67 & 0.56 & 0.48 & 0.32 \\
\hline & $\mathrm{PdAgBiSSe}_{2}$ & & $P 2_{1} 2_{1} 2_{1}$ & 0.593 & 4.824 & 8.353 & 14.231 & 1.12 & 1.01 & 1.81 & 0.33 & 0.68 & 0.89 \\
\hline & & & & & & & & 1.09 & 1.09 & 1.75 & 0.6 & 1.1 & 0.81 \\
\hline & $\mathrm{PdAgBiSeSSe}$ & & $P 2_{1} 2_{1} 2_{1}$ & 0.310 & 5.097 & 8.331 & 14.115 & 0.91 & 1.6 & 0.67 & 0.44 & 0.45 & 0.51 \\
\hline & $\mathrm{PdAgBiSe}_{2} \mathrm{~S}$ & & $P 2_{1} 2_{1} 2_{1}$ & 0.676 & 5.071 & 8.395 & 13.901 & 1.55 & 1.03 & 1.83 & 0.66 & 0.49 & 0.33 \\
\hline & & & & & & & & 1.38 & 0.94 & 1.54 & 0.92 & 0.65 & 0.54 \\
\hline & $\mathrm{PdAgBiS}_{2} \mathrm{Se}$ & & $P 2_{1} 2_{1} 2_{1}$ & 0.512 & 4.914 & 8.181 & 13.875 & 0.72 & 1.16 & 0.8 & 0.31 & 0.53 & 0.68 \\
\hline & $\mathrm{PdAgBiSeS}_{2}$ & & $P 2{ }_{1} 2_{1} 2_{1}$ & 0.564 & 5.096 & 8.256 & 13.733 & 1.05 & 0.91 & 2.11 & 0.43 & 0.91 & 1.37 \\
\hline & & & & & & & & 0.98 & 1.03 & 2.08 & 0.02 & 1.13 & 0.45 \\
\hline & PdAgBiSSeS & & $P 2{ }_{1} 2_{1} 2_{1}$ & 0.736 & 4.763 & 8.251 & 14.070 & 1.16 & 1.22 & 2.52 & 0.64 & 0.39 & 0.48 \\
\hline & & & & & & & & 1.02 & 1.15 & 2.13 & 0.7 & 0.45 & 0.5 \\
\hline
\end{tabular}




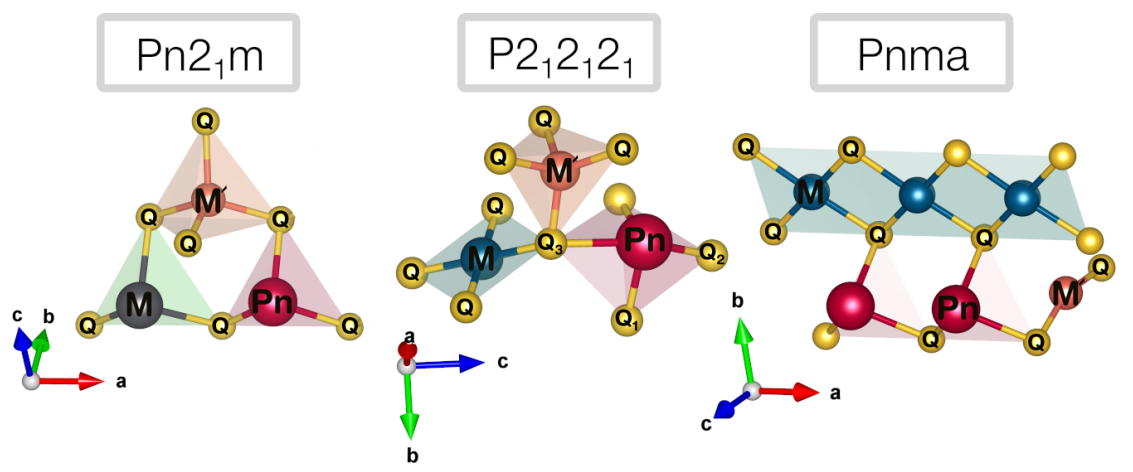

Table S 2: Bader Charge analysis and average bond length with calculated bond order. Note that the Bader charge of $Q$ constituents are averaged for simplicity $\left(M=\mathrm{Pd}, \mathrm{Pb}, \mathrm{Ni}, \mathrm{Pt} ; M^{\prime}=\mathrm{Cu}\right.$, $\mathrm{Ag} ; Q=\mathrm{Se}, \mathrm{S})$.

\begin{tabular}{|c|c|c|c|c|c|c|c|c|}
\hline \multirow{2}{*}{ Compounds } & \multicolumn{4}{|c|}{ Bader Charge } & \multicolumn{4}{|c|}{ Bond Length (ICOHP) } \\
\hline & $M$ & $M^{\prime}$ & $P n$ & $Q(a v g)$ & $M-Q$ & $M^{\prime}-Q$ & $P n-Q_{1}$ & $P n-Q_{3}$ \\
\hline $\mathrm{NiCuSbS}_{3}\left(P 2_{1} 2_{1} 2_{1}\right)$ & 0.43 & 0.5 & 1.07 & 0.66 & $2.23(1.2)$ & $2.29(\mathbf{0 . 6 0})$ & $2.57(\mathbf{1 . 2 0})$ & $2.88(0.7)$ \\
\hline $\mathrm{NiCuBiS}_{3}\left(P 2_{1} 2_{1} 2_{1}\right)$ & 0.40 & 0.49 & 1.08 & 0.65 & $2.26(\mathbf{1 . 2 0})$ & $2.29(\mathbf{0 . 7 0})$ & $2.68(1.30)$ & $2.91(\mathbf{0 . 8 0})$ \\
\hline $\mathrm{PtCuBiS}_{3}\left(P 2_{1} 2_{1} 2_{1}\right)$ & 0.06 & 0.49 & 1.13 & 0.55 & $2.36(\mathbf{1 . 2 0})$ & $2.29(\mathbf{0 . 7 0})$ & $2.73(\mathbf{1 . 2 0})$ & $2.89(\mathbf{0 . 9 0})$ \\
\hline $\mathrm{PdCuBiS}_{3}\left(P 2_{1} 2_{1} 2_{1}\right)$ & 0.00 & 0.36 & 0.92 & 0.42 & $2.35(\mathbf{0 . 5 0 )}$ & $2.29(\mathbf{0 . 7 0})$ & $2.69(1.20)$ & $2.89(\mathbf{0 . 8 0})$ \\
\hline $\mathrm{PdCuBiSe}_{3}\left(P 2_{1} 2_{1} 2_{1}\right)$ & 0.00 & 0.36 & 0.92 & 0.42 & $2.48(\mathbf{0 . 6 0 )}$ & $2.41(\mathbf{0 . 9 0 )}$ & $2.85(\mathbf{1 . 2 0})$ & $3.07(\mathbf{0 . 7 0})$ \\
\hline $\mathrm{PtCuBiSe}_{3}\left(P 2_{1} 2_{1} 2_{1}\right)$ & 0.19 & 0.35 & 0.94 & 0.37 & $2.49(\mathbf{1 . 5 0})$ & $2.38(\mathbf{0 . 9 0 )}$ & $2.91(\mathbf{1 . 2 0})$ & $3.1(\mathbf{0 . 7 )}$ \\
\hline $\mathrm{NiCuBiSe}_{3}\left(P 2_{1} 2_{1} 2_{1}\right)$ & 0.22 & 0.36 & 0.87 & 0.48 & $2.37(\mathbf{1 . 4 0 )}$ & $2.40(\mathbf{0 . 9 0})$ & $2.85(\mathbf{1 . 2 0})$ & $3.1(\mathbf{0 . 7})$ \\
\hline $\mathrm{PdCuBiSSe}_{2}\left(P 2_{1} 2_{1} 2_{1}\right)$ & 0.04 & 0.44 & 0.98 & 0.48 & $2.46(\mathbf{0 . 6 0 )}$ & $2.45(\mathbf{0 . 5})$ & $2.80(\mathbf{1 . 3})$ & $3.03(\mathbf{0 . 7})$ \\
\hline $\mathrm{PdCuBiS}_{2} \mathrm{Se}\left(P 2_{1} 2_{1} 2_{1}\right)$ & 0.10 & 0.46 & 1.06 & 0.54 & $2.37(\mathbf{0 . 5 0 )}$ & $2.3(\mathbf{0 . 7 0})$ & $2.70(\mathbf{1 . 2 0})$ & $2.99(\mathbf{0 . 8})$ \\
\hline $\mathrm{PdCuBiSeS}_{2}\left(P 2_{1} 2_{1} 2_{1}\right)$ & 0.14 & 0.43 & 1.06 & 0.54 & $2.38(\mathbf{0 . 5 0 )}$ & $2.42(\mathbf{0 . 7 0})$ & $2.80(\mathbf{1 . 3 0})$ & $2.92(\mathbf{0 . 8 0})$ \\
\hline $\mathrm{PdCuBiSe}_{2} \mathrm{~S}\left(P 2_{1} 2_{1} 2_{1}\right)$ & 0.05 & 0.39 & 1.00 & 0.51 & $2.48(\mathbf{0 . 6 0 )}$ & $2.45(\mathbf{0 . 5 0 )}$ & $2.85(\mathbf{1 . 2 0})$ & $2.96(\mathbf{0 . 7 0})$ \\
\hline $\mathrm{PdAgBiSe}_{3}\left(P 2_{1} 2_{1} 2_{1}\right)$ & 0.03 & 0.26 & 0.94 & 0.40 & $2.48(\mathbf{0 . 6})$ & $2.65(0.7)$ & $2.80(\mathbf{1 . 3})$ & $3.21(\mathbf{0 . 5})$ \\
\hline $\mathrm{PdAgBiSSe}_{2}\left(P 2_{1} 2_{1} 2_{1}\right)$ & 0.07 & 0.32 & 1.01 & 0.46 & $2.49(\mathbf{0 . 5 0})$ & $2.76(\mathbf{0 . 5 0 )}$ & $2.77(1.40)$ & $2.86(\mathbf{1 . 2 0})$ \\
\hline PdAgBiSSeS $\left(P 2_{1} 2_{1} 2_{1}\right)$ & 0.12 & 0.35 & 1.11 & 0.49 & $2.38(\mathbf{0 . 5})$ & $2.55(\mathbf{0 . 6 0 )}$ & $2.69(1.20)$ & $3.01(\mathbf{0 . 6})$ \\
\hline $\mathrm{PdAgBiSeS}_{2}\left(P 2_{1} 2_{1} 2_{1}\right)$ & 0.17 & 0.31 & 1.07 & 0.52 & $2.39(\mathbf{0 . 5})$ & $2.65(\mathbf{0 . 6 0 )}$ & $2.75(\mathbf{1 . 5 0})$ & $3.02(\mathbf{0 . 7})$ \\
\hline \multirow[t]{2}{*}{$\mathrm{PdAgBiSe}_{2} \mathrm{~S}\left(P 2_{1} 2_{1} 2_{1}\right)$} & 0.07 & 0.28 & 1.01 & 0.46 & $2.46(\mathbf{0 . 3 0})$ & $2.6(\mathbf{0 . 6 0 )}$ & $2.83(0.7)$ & $3.12(0.4)$ \\
\hline & $M$ & $M^{\prime}$ & $P n$ & $Q(a v g)$ & $M-Q$ & $M^{\prime}-Q$ & $P n-Q$ & \\
\hline $\mathrm{PbCuSbS}_{3}\left(\operatorname{Pn} 2_{1} m\right)$ & 0.97 & 0.44 & 1.08 & 0.83 & $2.85(\mathbf{1 . 3 0})$ & $2.34(\mathbf{0 . 6 0 )}$ & $2.49(\mathbf{2 . 1 0})$ & \\
\hline $\mathrm{PbCuAsS}_{3}\left(P n 2_{1} m\right)$ & 0.99 & 0.45 & 0.76 & 0.75 & $2.85(1.1)$ & $2.33(\mathbf{0 . 7 0})$ & $2.33(\mathbf{1 . 5})$ & \\
\hline $\mathrm{PbCuBiS}_{3}\left(P n 2_{1} m\right)$ & 0.97 & 0.43 & 1.1 & 0.83 & $2.86(1.20)$ & $2.34(\mathbf{0 . 6 0 )}$ & $2.60(1.6)$ & \\
\hline $\mathrm{PbCuBiSe}_{3}\left(P n 2_{1} m\right)$ & 0.84 & 0.3 & 0.91 & 0.76 & $2.99(\mathbf{1 . 2 0})$ & $2.46(0.70)$ & $2.76(1.60)$ & \\
\hline $\mathrm{PbCuBiS}_{3}($ Pnma $)$ & 3.88 & 1.18 & -5.50 & 0.6 & $2.99(\mathbf{0 . 9 0})$ & $2.39(\mathbf{0 . 5 0})$ & $2.7(1.1)$ & \\
\hline $\mathrm{PbCuBiSe}_{3}($ Pnma $)$ & 0.87 & 0.3 & 1.02 & 0.72 & $3.14(1)$ & $2.49(\mathbf{0 . 6})$ & $2.93(1.1)$ & \\
\hline $\mathrm{PdCuBiSe}_{3}($ Pnma $)$ & 0.03 & 0.37 & 0.94 & 0.45 & $2.53(0.5)$ & $2.34(1)$ & $2.79(1.3)$ & \\
\hline $\mathrm{PdCuBiS}_{3}($ Pnma $)$ & 0.22 & 0.47 & 1.16 & 0.62 & $2.39(\mathbf{0 . 5 0})$ & $2.17(0.90)$ & $2.64(1.40)$ & \\
\hline
\end{tabular}



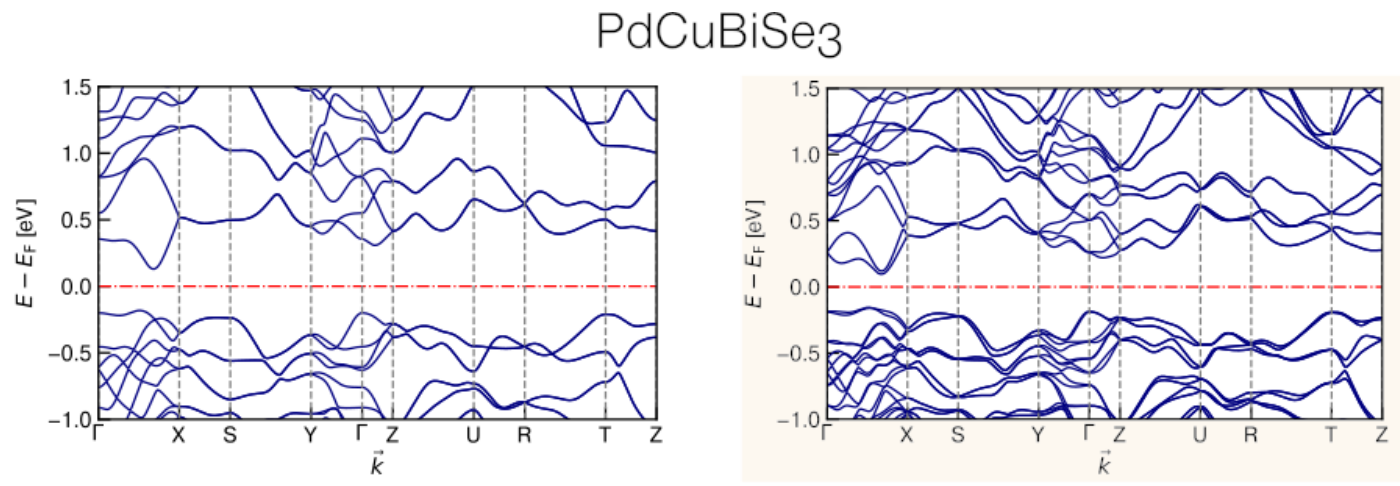

$\mathrm{NiCuSbS}_{3}$
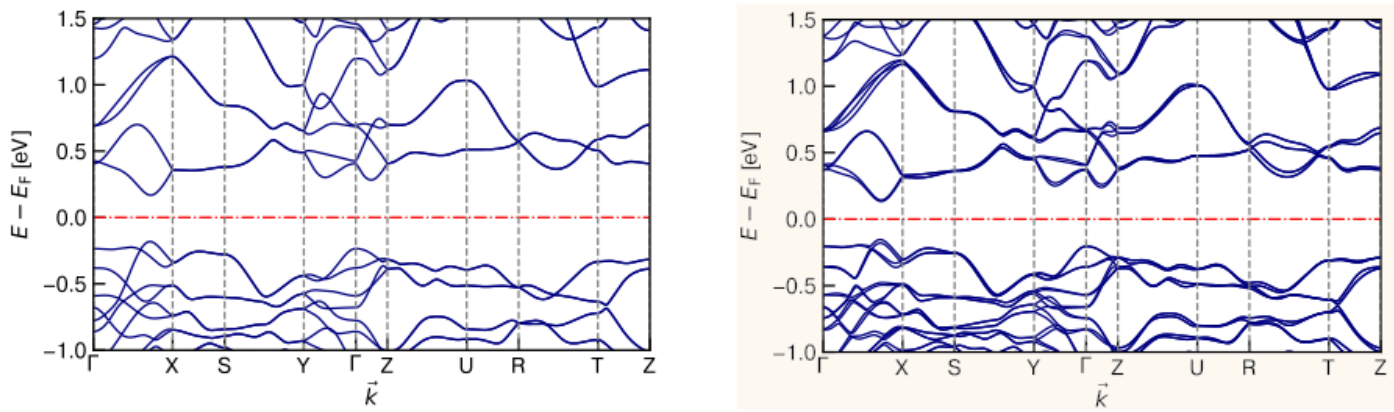

$\mathrm{PtCuBiS}_{3}$
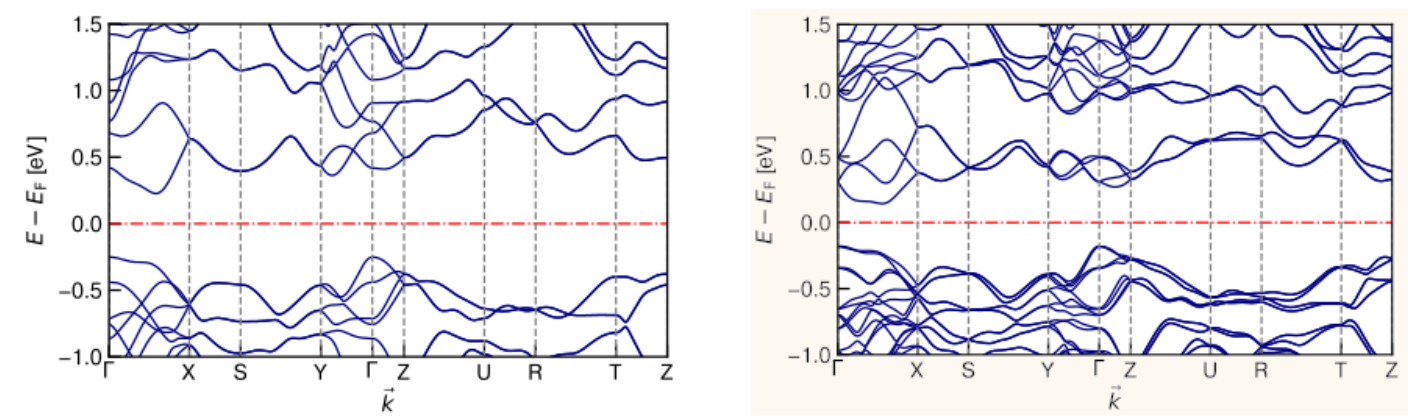

Fig. S 2: Band structures of $\mathrm{PdCuBiSe}_{3}, \mathrm{NiCuSbS}_{3}$ and $\mathrm{PtCuBiS}_{3}$ with and without Spin-Orbit Coupling(shaded area refer to SOC). 


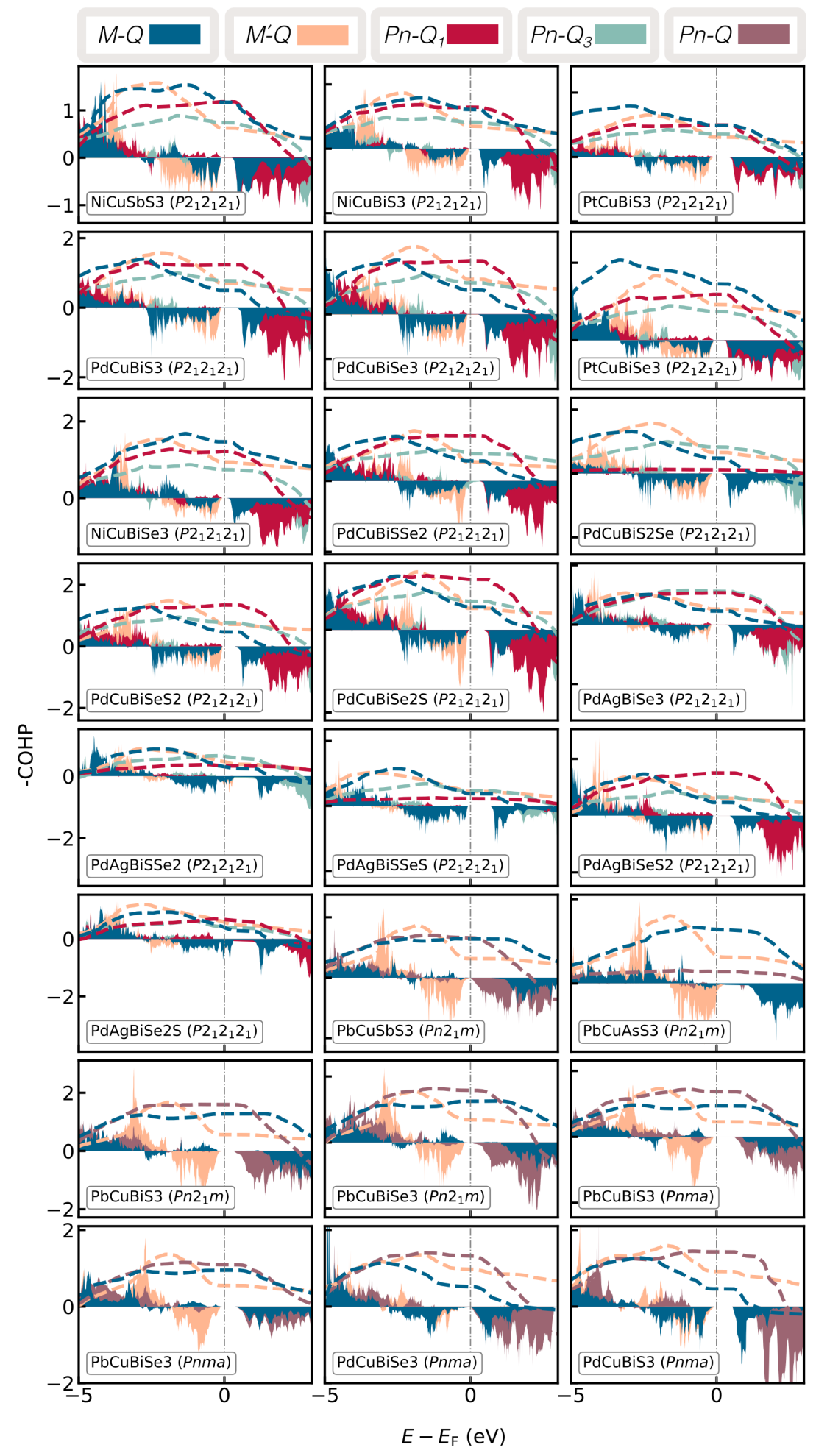

Fig. S3: Integrated Crystal Orbital Hamilton Population(ICOHP) of lapieites, bournonites, and substituted compounds of these main groups. The rise in anti-bonding characteristic of $\mathrm{Cu}-Q$ bond results in softer bond. 


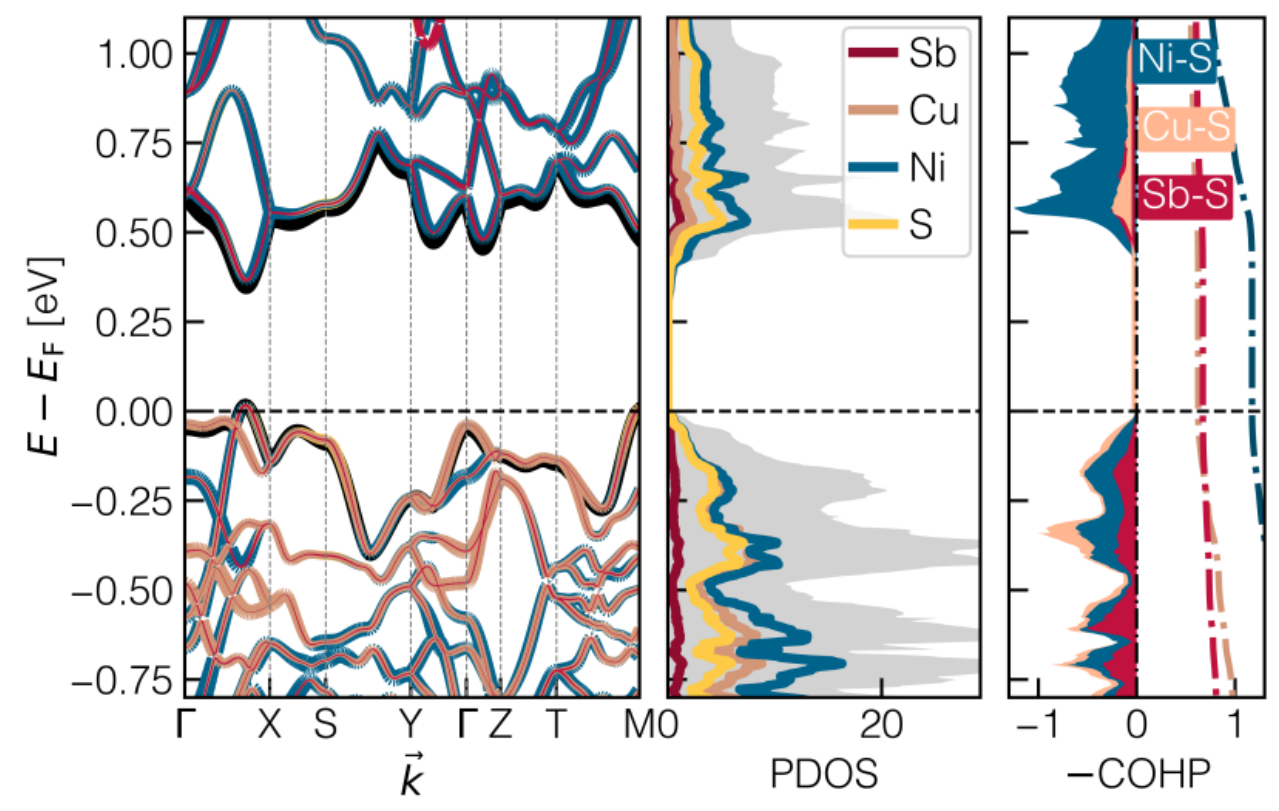

Fig. S 4: Electronic band structure of $\mathrm{NiCuSbS}_{3}$ projected on each element(left) with partial density of states and COHP analysis(right). The black lines behind valence and conduction bands are the calculated band structure with including SOC. It is obvious that SOC does not change the overall shape of these main bands.
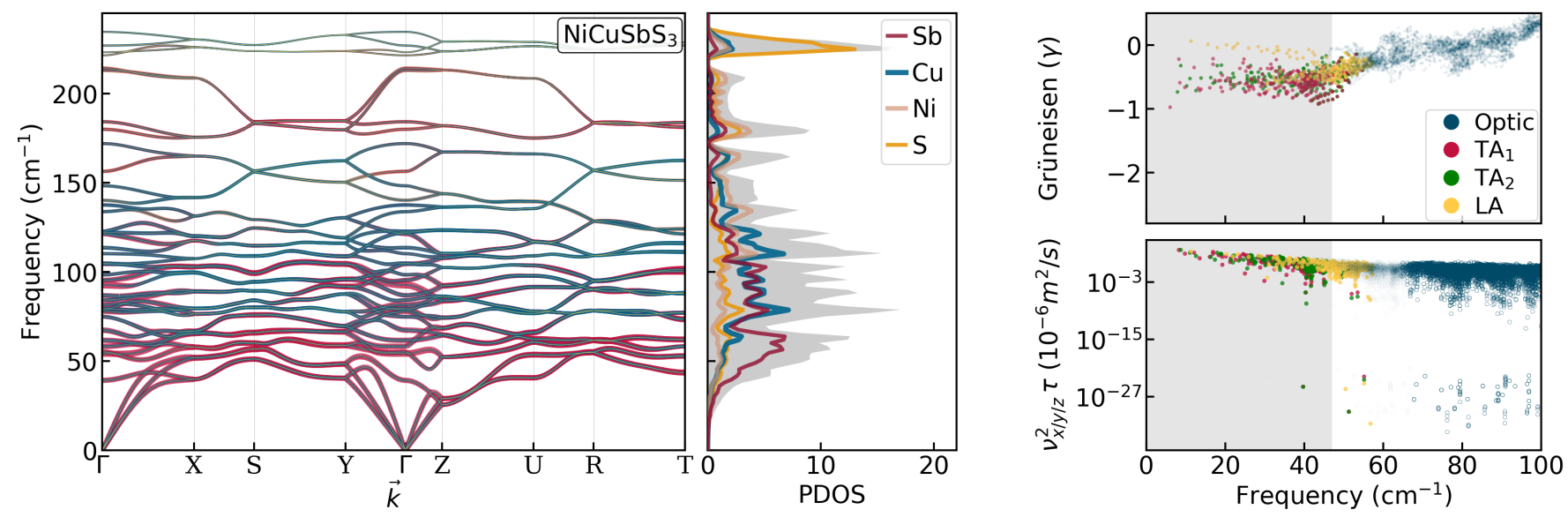

Fig. S5: The atom-projected phonon dispersion and its density of states, mode Grüneisen parameters and the square of group velocities multiplied by the mean free path for $\mathrm{NiCuSbS}_{3}$. 


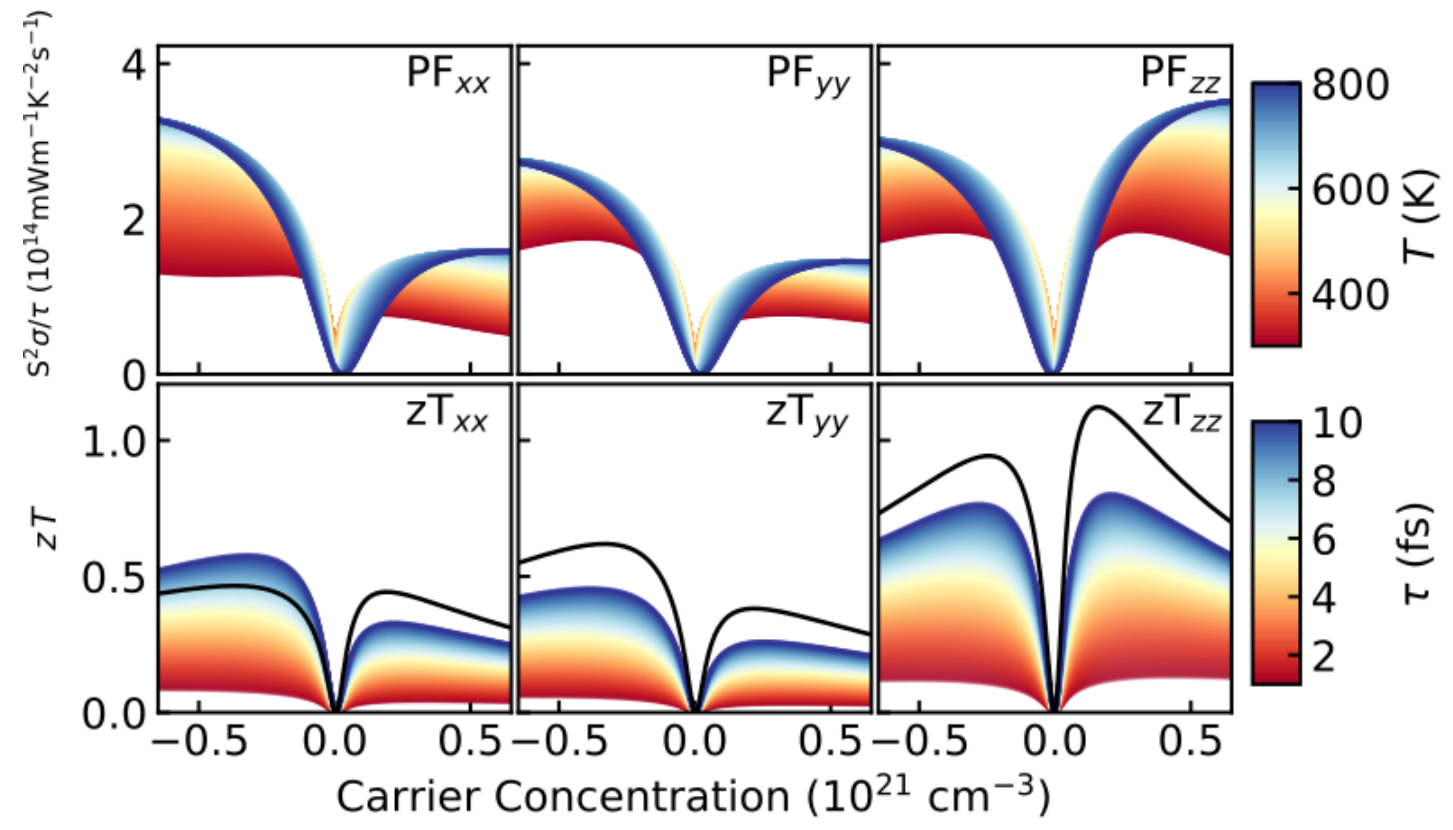

Fig. S6: Figure of merit and power factor variations with respect to temperature for $\mathrm{NiCuSbS}_{3}$. The black line indicates the calculated $z T$ with relaxation time via deformation potential approximation. The value of $z T$ bypass one at $600 \mathrm{~K}$ which makes $\mathrm{NiCuSbS}_{3}$ a promising $\mathrm{TE}$ candidate. 


\section{$\mathrm{PdCuBiSe}_{3}$}

a)

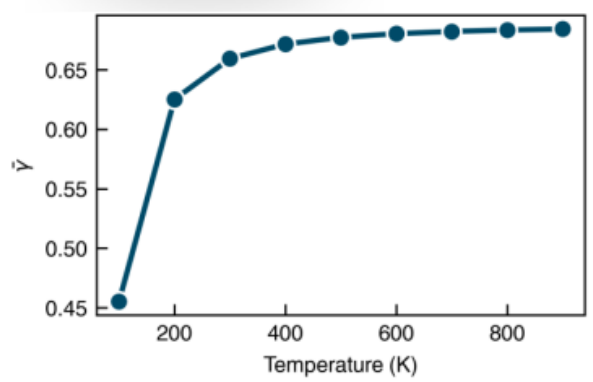

b)

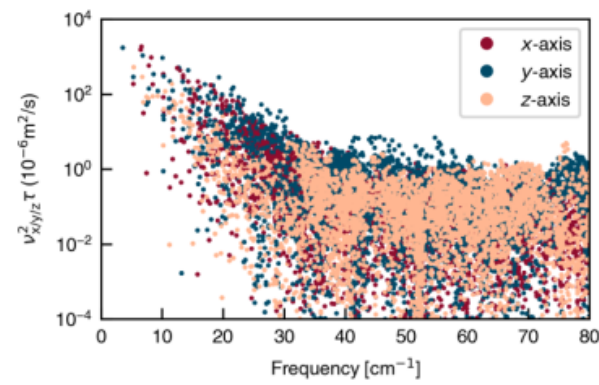

C)

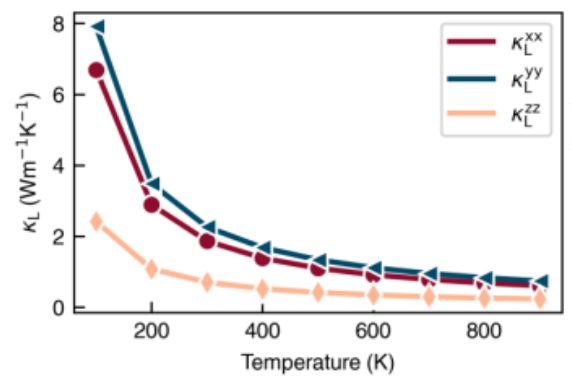

d)

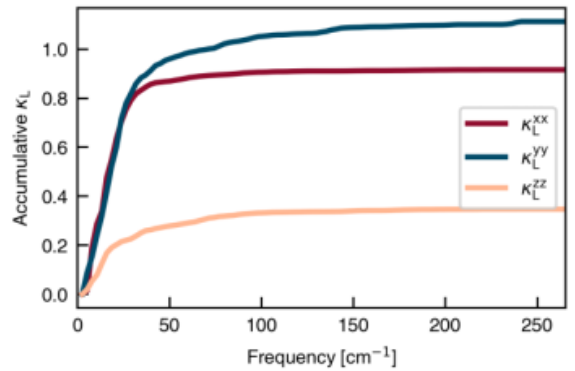

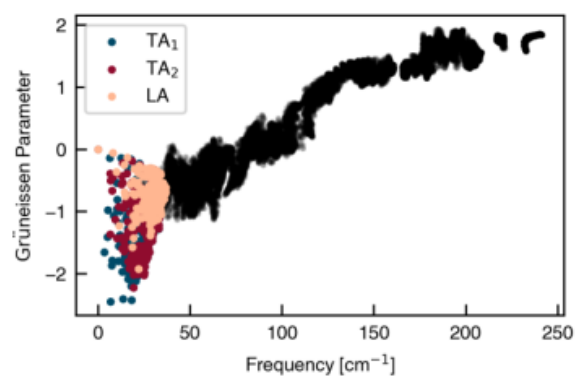
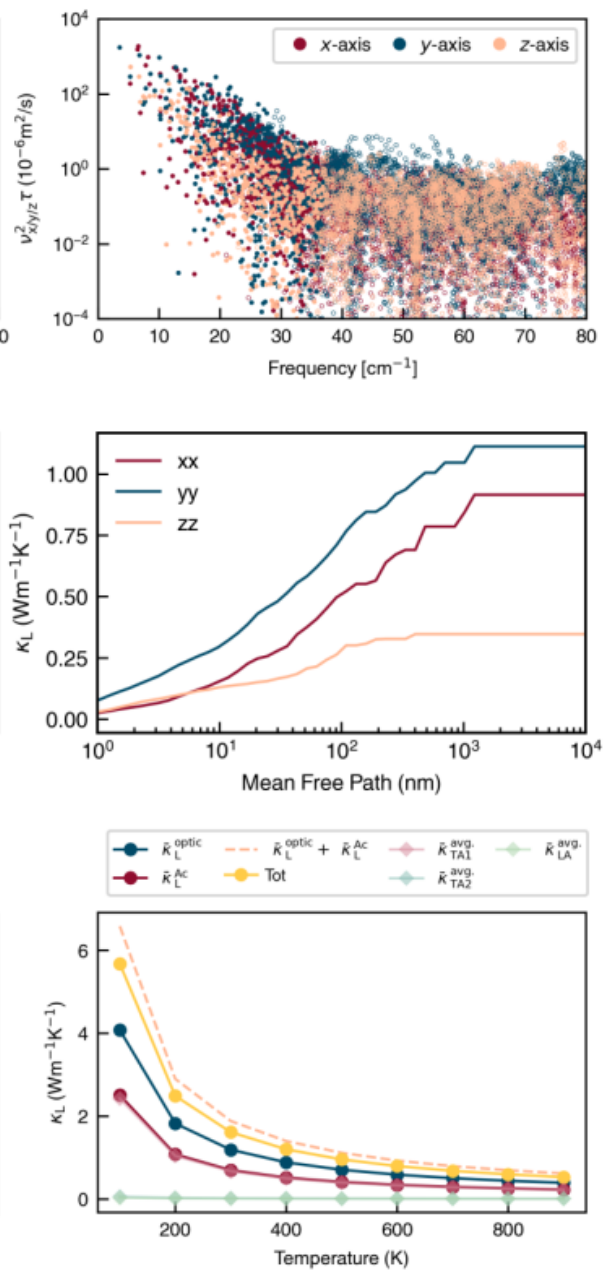

Fig.S 7: (a) Grüneisen and mode projected Grüneisen parameters, (b) The squared of group velocities multiplied by the mean free path, (c) $\kappa_{\mathrm{L}}$ and accumulated $\kappa_{\mathrm{L}}$ with respect to mean free path, (d) Accumulated $\kappa_{\mathrm{L}}$ with respect to frequency and optical contributions, acoustic contributions in $\kappa_{\mathrm{L}}$ and its average, with respect to temperature for $\mathrm{PdCuBiSe}$. 


\section{$\mathrm{NiCuSbS}_{3}$}

a)
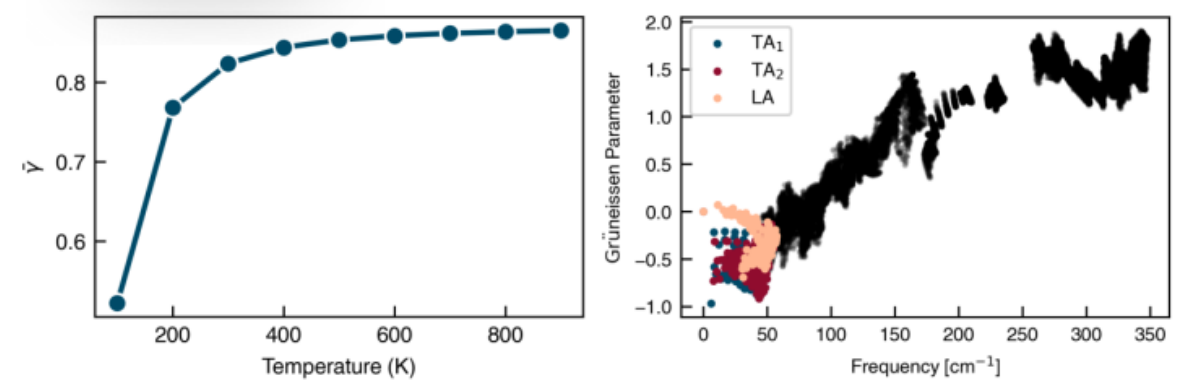

b)
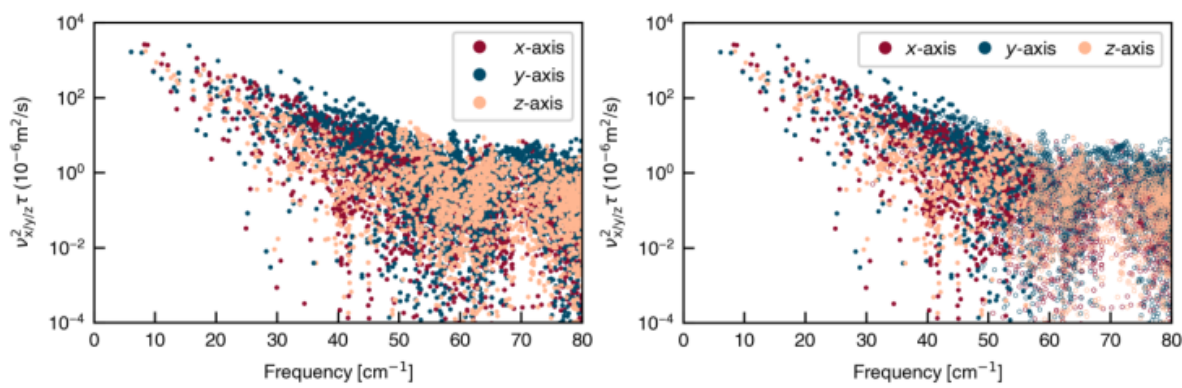

C)
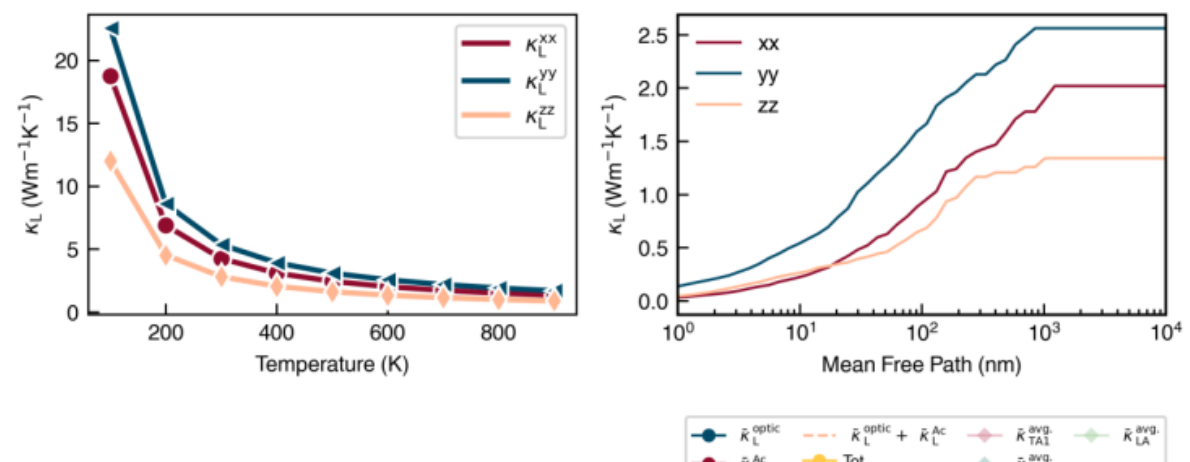

d)
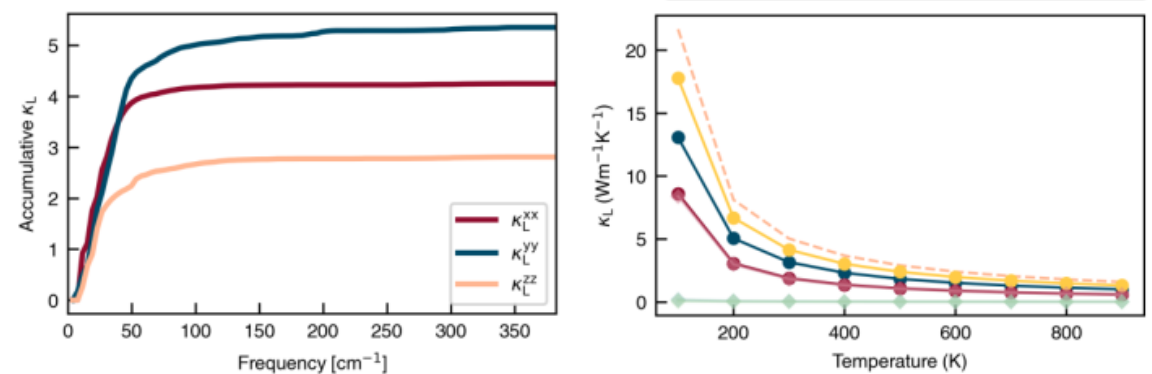

Fig. S 8: (a) Grüneisen parameter and mode projected Grüneisen parameter, (b) The squared of group velocities multiplied by the mean free path, (c) $\kappa_{\mathrm{L}}$ and accumulated $\kappa_{\mathrm{L}}$ with respect to mean free path, (d) Accumulated $\kappa_{\mathrm{L}}$ with respect to frequency and optical contributions, acoustic contributions in $\kappa_{\mathrm{L}}$ and its average, with respect to temperature for $\mathrm{NiCuSbS}_{3}$. 
Table S 3: Infinite dielectric constant $\left(\epsilon_{\infty}\right)$, Band Gap,(BG) with HSE exchange-correlation functional and Born Effective charge $\left(Z^{*}\right)$ of main lapieites and their bournonites counterparts. It has been shown that lapieites have relatively higher dielectric constant and also higher $Z^{*}$ than bournonites.

\begin{tabular}{lcccccc}
\hline \multirow{2}{*}{ Compounds } & \multirow{2}{*}{$\epsilon_{\infty}^{H S E}(\mathbf{G G A})$} & $\mathrm{BG}(\mathrm{eV})^{H S E}$ & \multicolumn{3}{c}{$Z^{*}$} \\
\cline { 5 - 8 } & $16.19(\mathbf{3 0 . 5 9})$ & 0.95 & 1.19 & 0.98 & 4.83 & 1.51 \\
$\mathrm{PdCuBiSe}_{3}\left(P 2_{1} 2_{1} 2_{1}\right)$ & $10.59(\mathbf{1 4 . 6 4})$ & 0.83 & 3.97 & 0.78 & 3.78 & 2.4 \\
$\mathrm{PbCuBiSe}_{3}\left(P 2_{1} 2_{1} 2_{1}\right)$ & $11.46(\mathbf{2 4 . 7 6})$ & 1.4 & 0.79 & 0.95 & 4.67 & 1.59 \\
$\mathrm{NiCuSbS}_{3}\left(P 2_{1} 2_{1} 2_{1}\right)$ & $7.88(\mathbf{9 . 8 5})$ & 1.6 & 3.8 & 0.5 & 3.1 & 2.1 \\
$\mathrm{PbCuSbS}_{3}\left(P 2_{1} 2_{1} 2_{1}\right)$ & & &
\end{tabular}
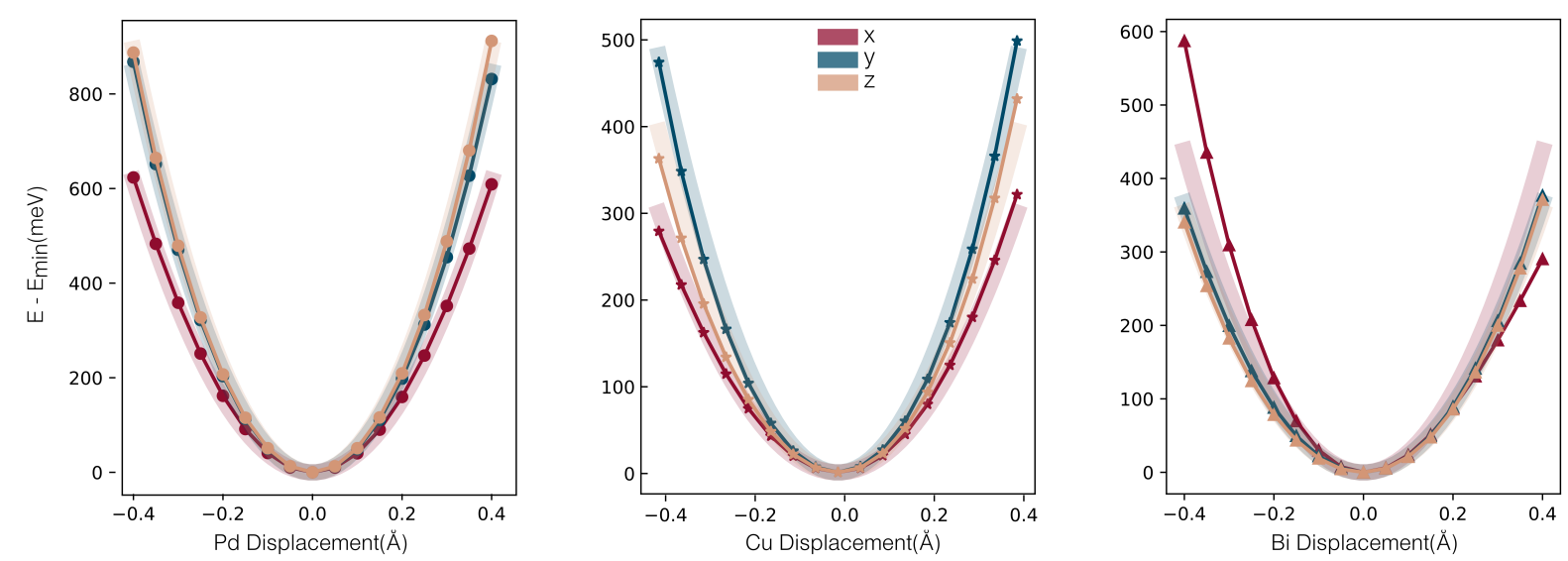

Fig. S 9: Energy vs displacement of $\mathrm{PdCuBiSe}_{3}$ cations along different crystallographic axis. Except for $\mathrm{Bi}$, the potential energy behavior with respect to change of atomic displacement is harmonic. 
Table S 4: Energy (meV) of three valence band maximums (VBM) and flat band for lapieites. Note that the alignment of flat band does not affect the hole-doped power factor.

\begin{tabular}{lccccc}
\hline & $\Delta E_{V B 1}$ & $\Delta E_{V B 2}$ & $\Delta E_{V B 3}$ & $\Delta E_{V B 4}$ & $\Delta E_{\text {Flat }}$ \\
\hline $\mathrm{PdCuBiSe}_{3}\left(P 2_{1} 2_{1} 2_{1}\right)$ & 0.0 & 18.5 & 29.0 & 7.8 & 55.9 \\
$\mathrm{PdCuBiS}_{3}\left(P 2_{1} 2_{1} 2_{1}\right)$ & 7.7 & 2.0 & 65.64 & 51.9 & 117.1 \\
$\mathrm{PtCuBiS}_{3}\left(P 2_{1} 2_{1} 2_{1}\right)$ & 47.1 & 0.0 & 147.9 & 150.2 & 205.8 \\
$\mathrm{NiCuBiS}_{3}\left(P 2_{1} 2_{1} 2_{1}\right)$ & 12.5 & 0.0 & 151.2 & 91.0 & 118.1 \\
$\mathrm{NiCuSbS}_{3}\left(P 2_{1} 2_{1} 2_{1}\right)$ & 44.8 & 53.1 & 152.9 & 7.97 & 96.8 \\
\hline
\end{tabular}

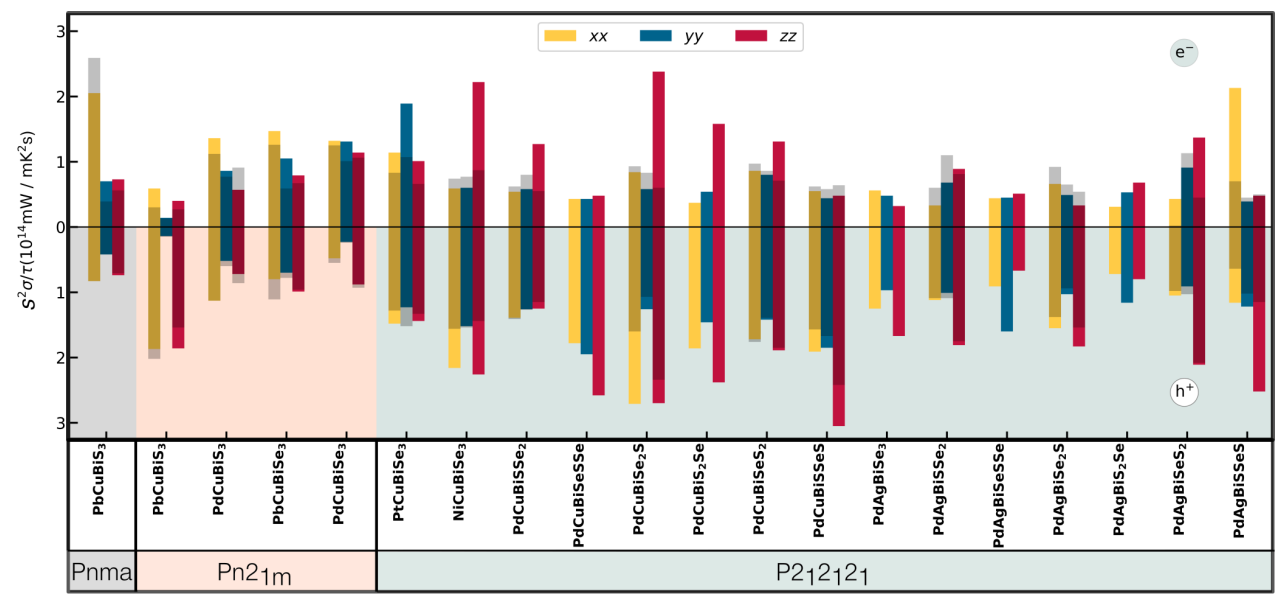

Fig. S 10: Calculated power factors of bournonites and substituted lapieites. This plot demonstrates the comparative power factors of these compounds. Also, it can be seen that substituted compounds are generally better hole-doped thermoelectric materials, stemmed from their main origins. 


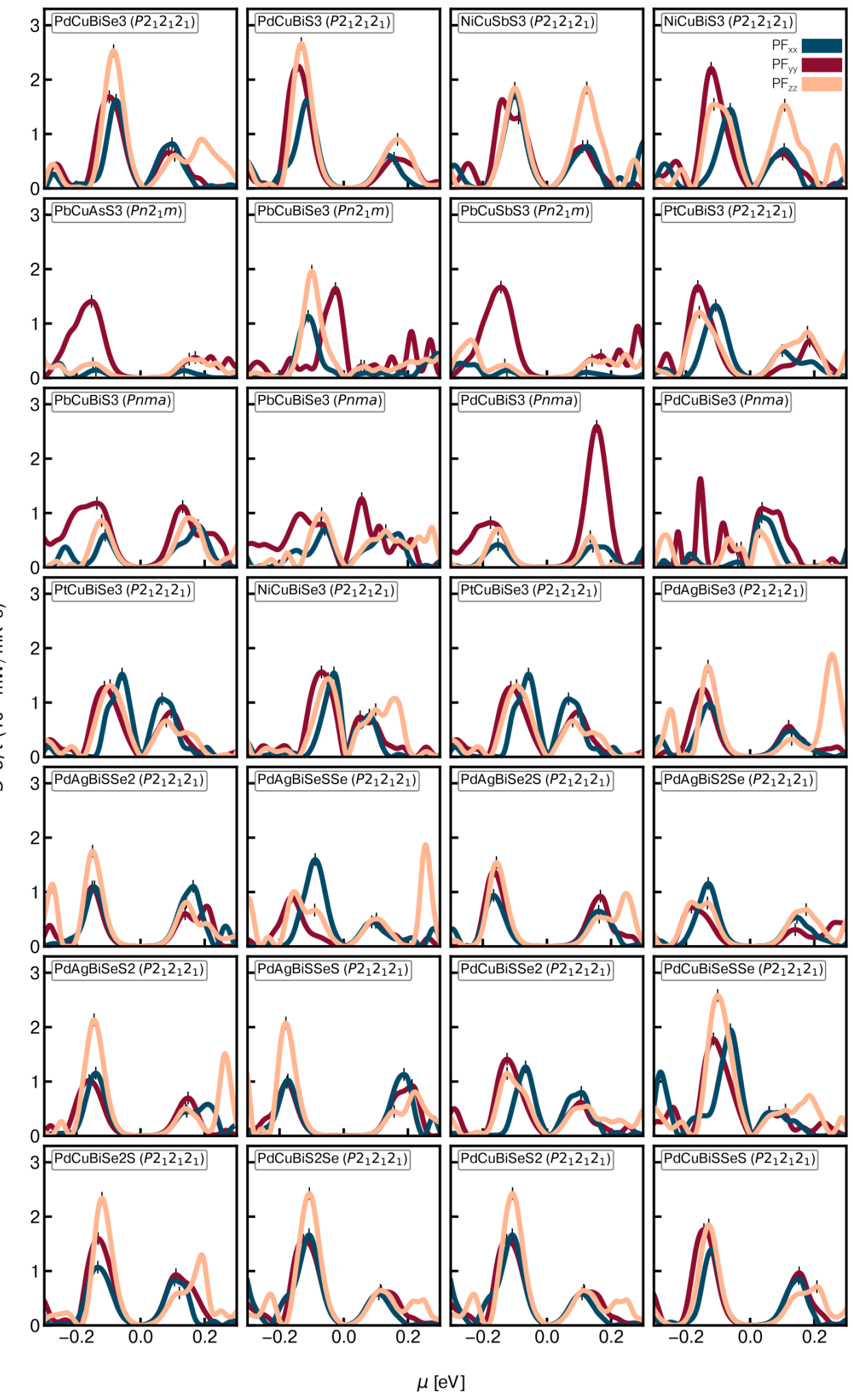

Fig. S11: Calculated power factors with respect to chemical potential. 

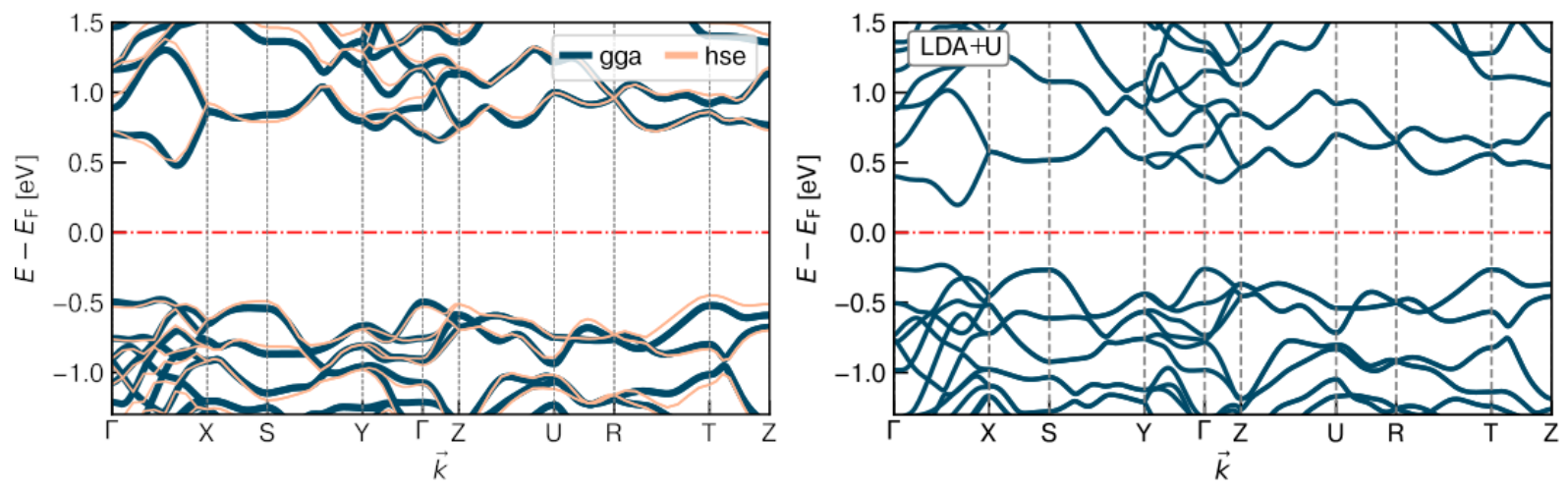

Fig. S 12: Band gap for $\mathrm{PdCuBiSe}_{3}$ with $\mathrm{LDA}+\mathrm{U}$ and HSE exchange-correlation functionals. It can be seen from the band structures that neither of these approximations change the overall shape of band structure but both can make the gap larger. $\mathrm{U}$ has been applied for $\mathrm{Cu}$ atom about $4 \mathrm{eV}$.

Table S 5: DFT calculated delocalization index $(D I)$, electron transfer $(E T)$ and integrated $\mathrm{COHP}(\mathrm{ICOHP})$ in $\mathrm{PbCuBiSe}_{3}\left(P n 2_{1} m\right)$ and $\mathrm{PbCuSbS}_{3}\left(P n 2_{1} m\right)$.

\begin{tabular}{lcccc}
\hline & Analysis & $M-Q$ & $\mathrm{Cu}-Q$ & $P n-Q$ \\
\hline & & & & \\
$\mathbf{P b C u B i S e}_{3}\left(P n 2_{1} m\right)$ & $D I$ & 0.52 & 0.60 & 0.63 \\
& $E T$ & 0.9 & 0.18 & 1.4 \\
& ICOHP & 1.3 & 0.75 & 1.25 \\
& & & \\
$\mathbf{P b C u S b S}_{3}\left(\operatorname{Pn} 2_{1} m\right)$ & $D I$ & 0.40 & 0.61 & 0.79 \\
& $E T$ & 0.5 & 0.32 & 0.5 \\
& ICOHP & 1.3 & 0.75 & 2 \\
\hline
\end{tabular}



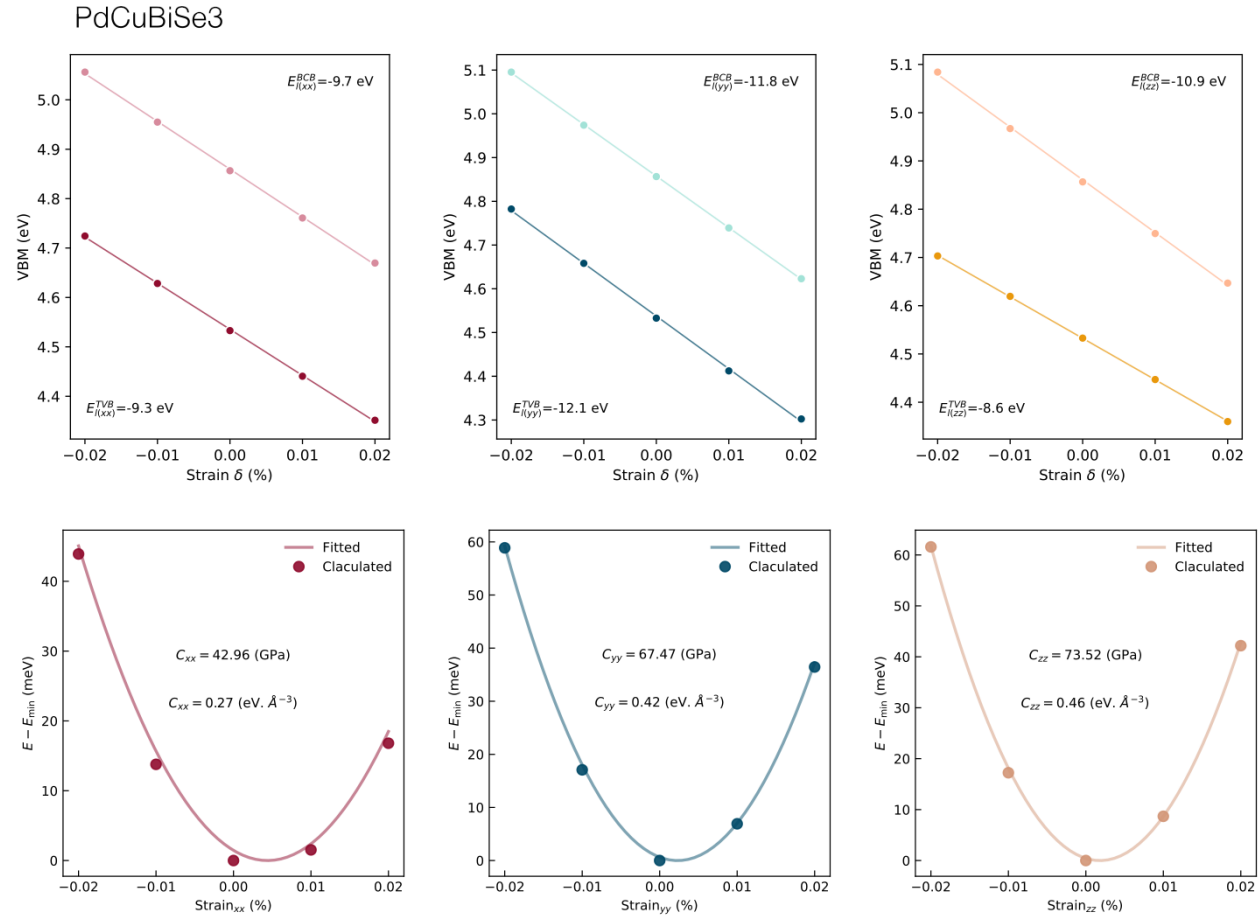

$\mathrm{NiCuSbS3}$
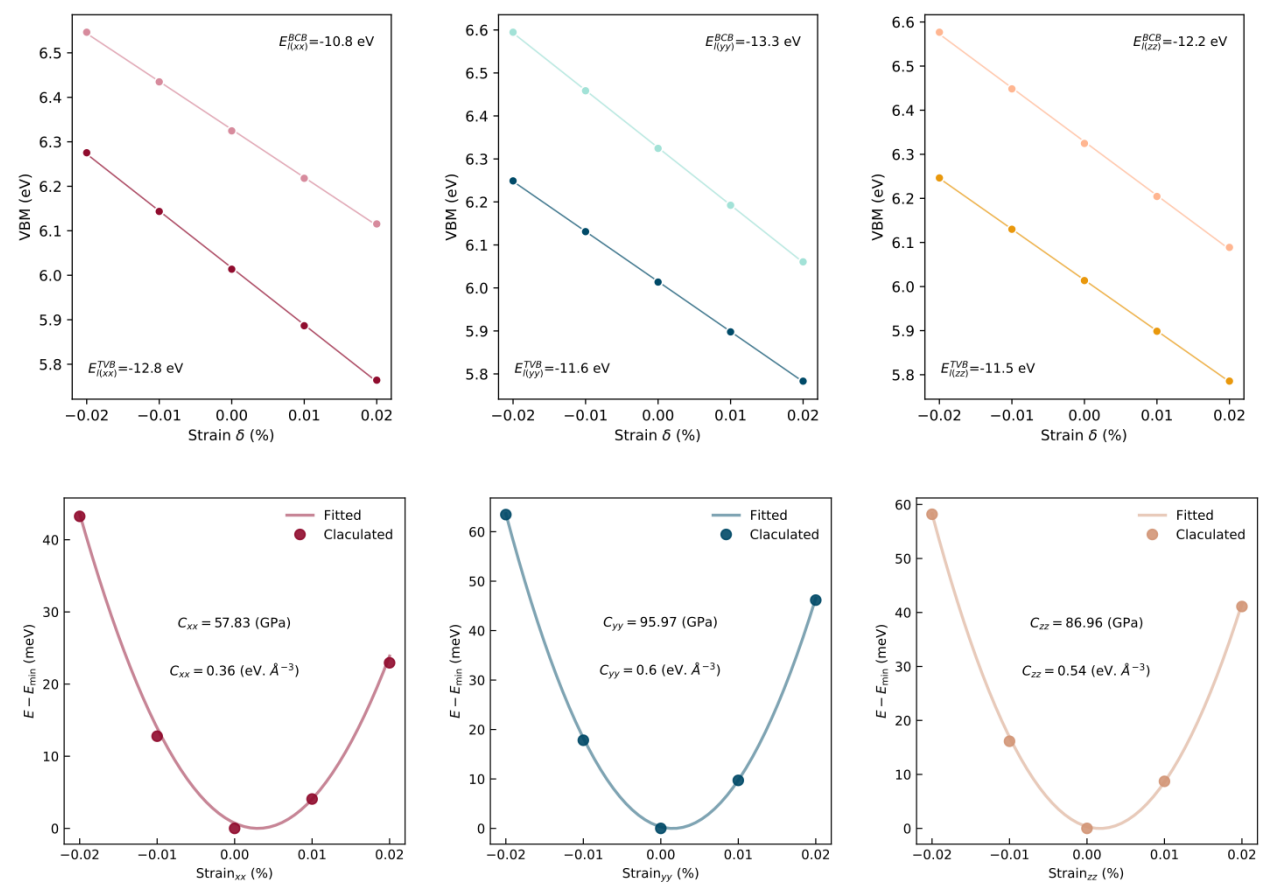

Fig. S13: Deformation potential and elastic moduli for $\mathrm{PdCuBiSe}_{3}$ and $\mathrm{NiCuSbS}_{3}$. 

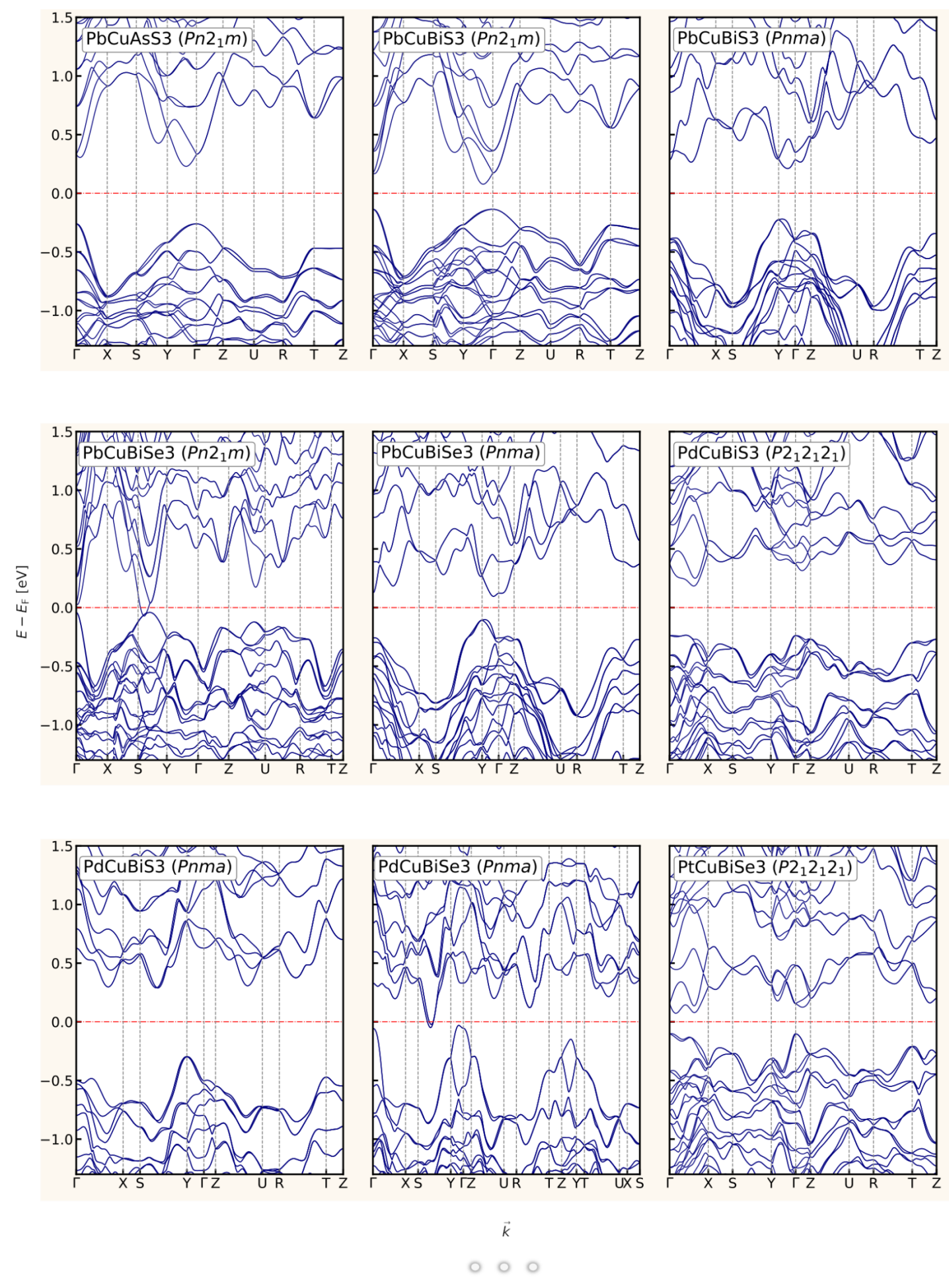
o 00
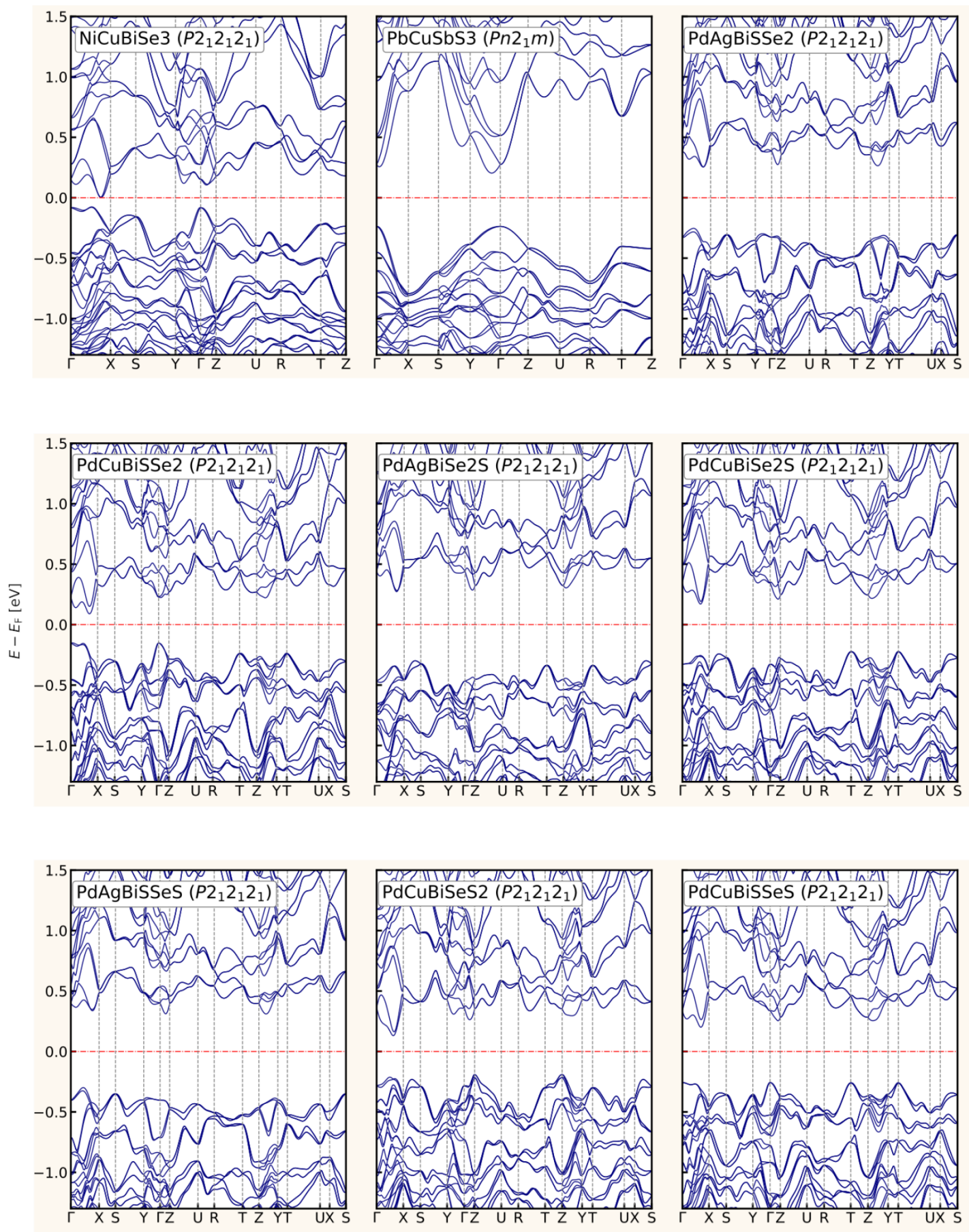

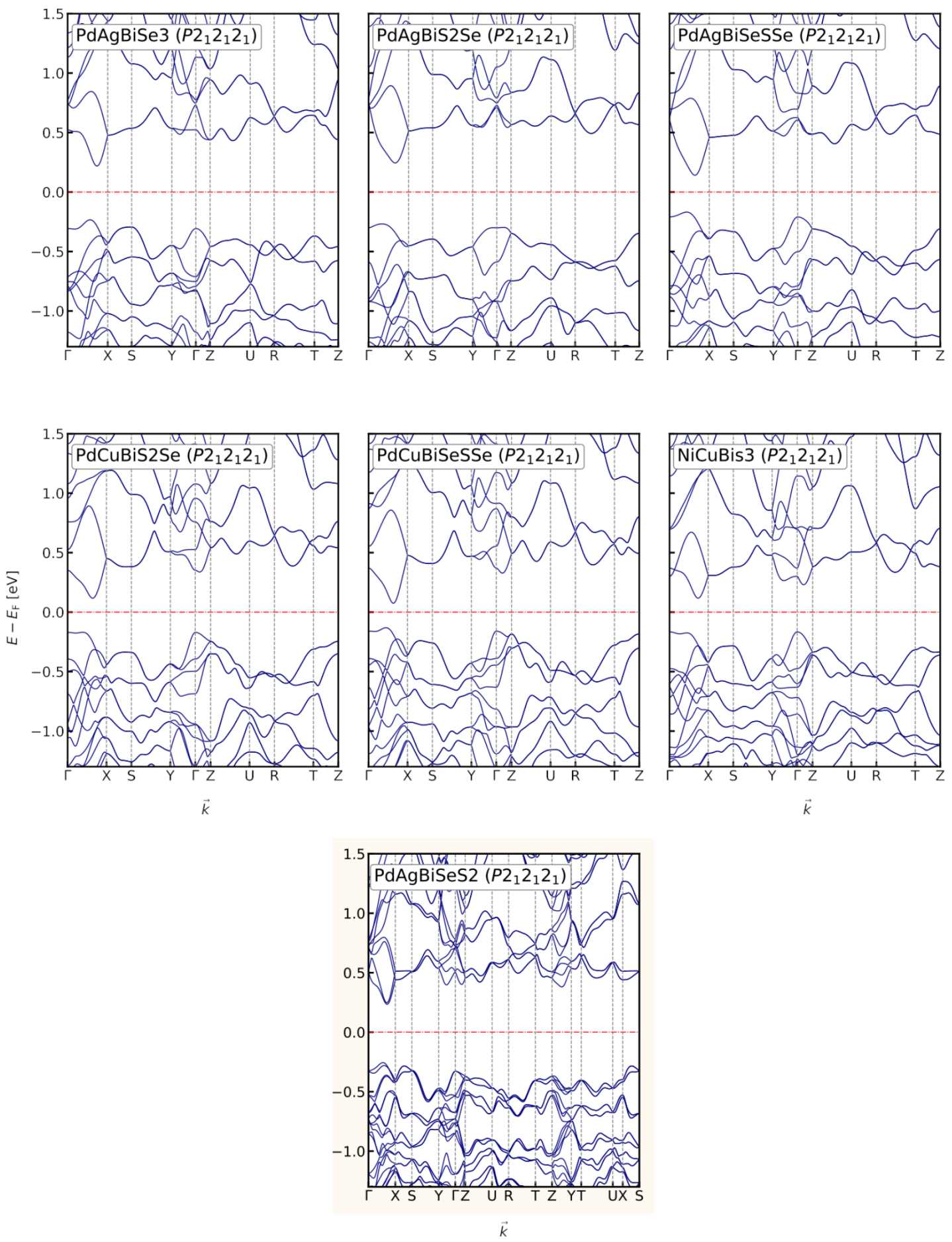

Fig. S14: Electronic band structures of compounds (shaded area refer to SOC). 

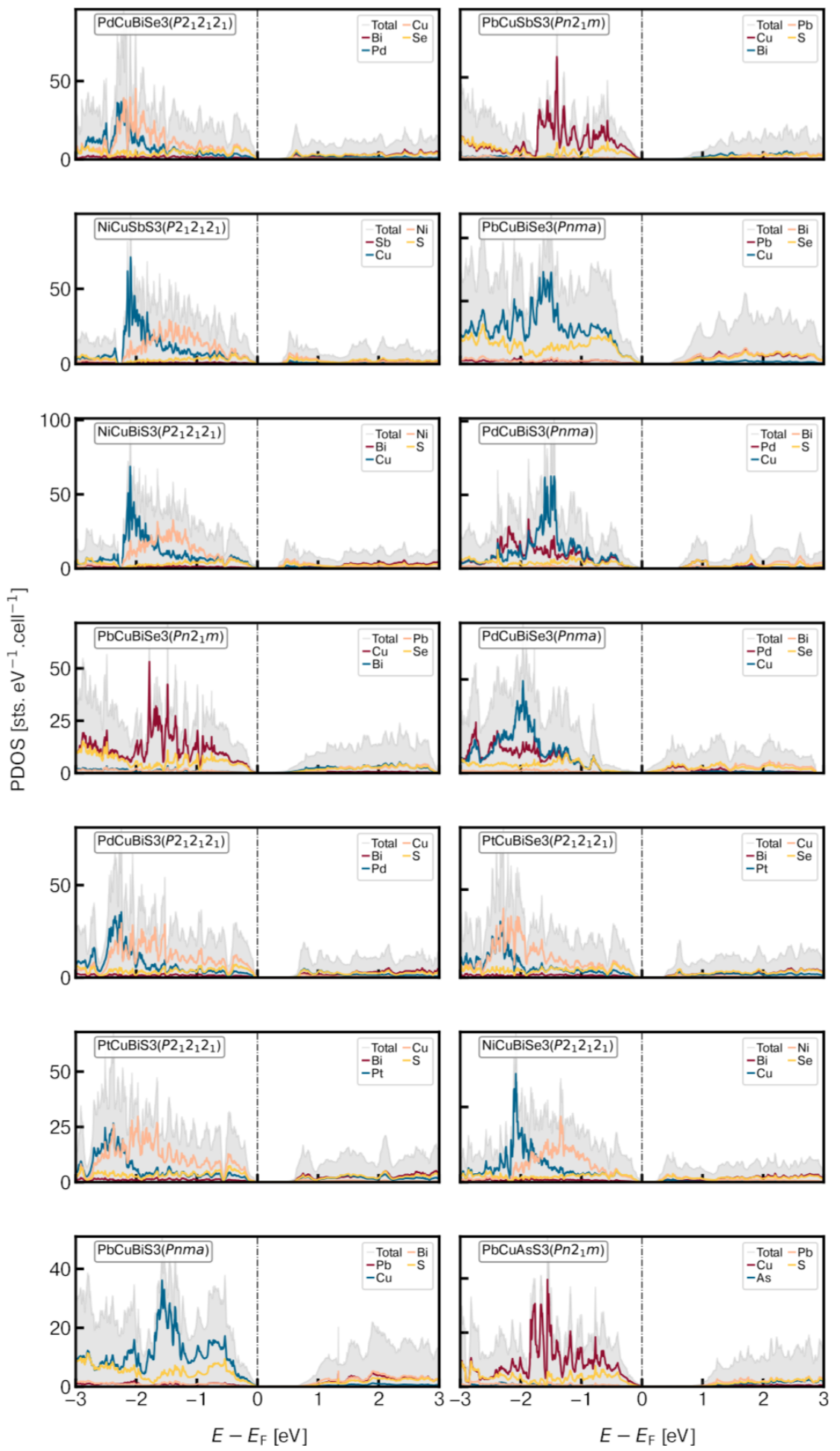

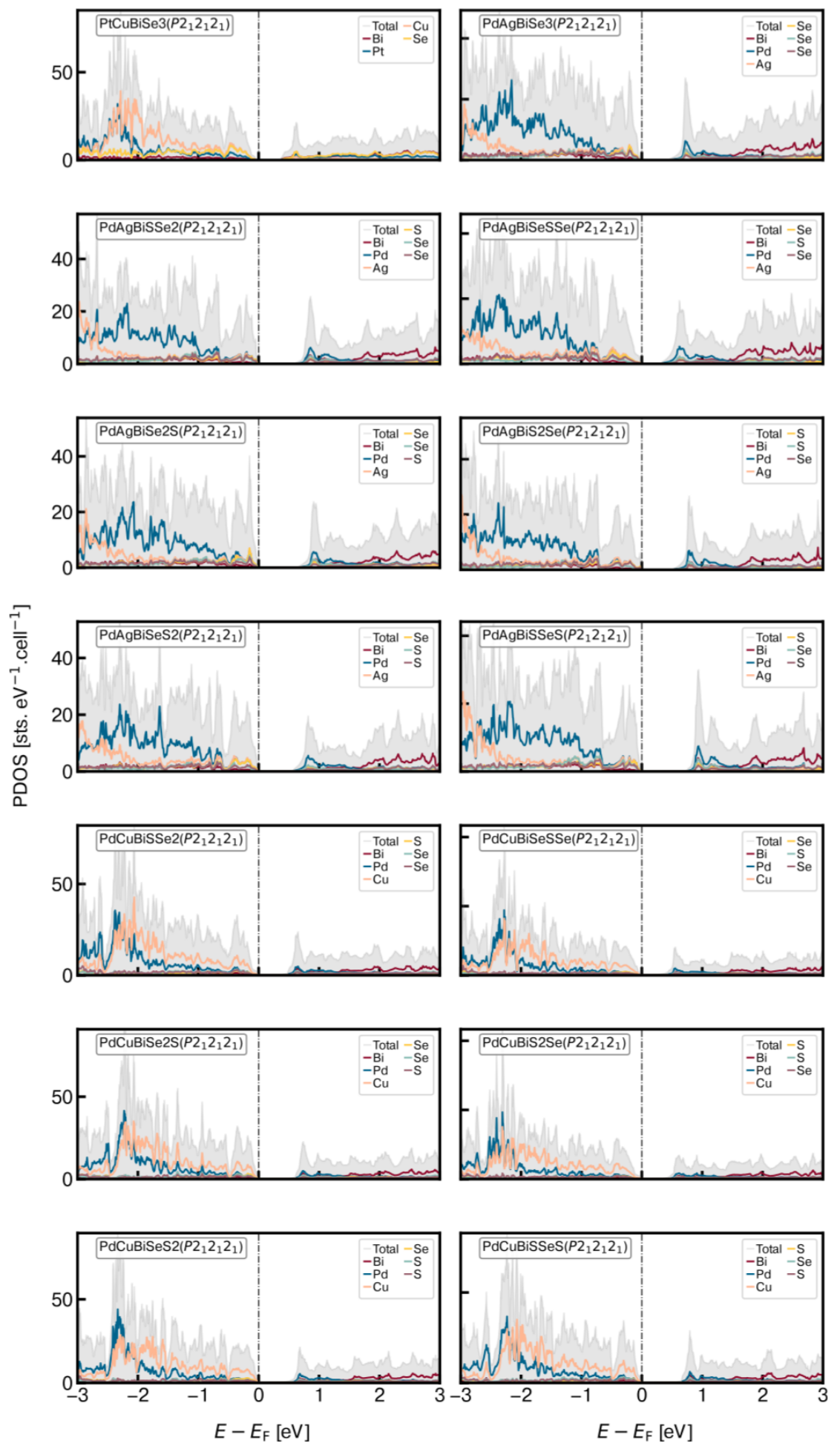

Fig. S15: Atom projected density of states for compounds. 